\title{
Spontaneous Broad Ligament Hematoma after Vaginal Delivery Requiring Hysterectomy
}

\author{
Megan Varvoutis, MD ${ }^{1}$ Nguyen Thao Thi Nguyen, BA ${ }^{2}$ Chad Grotegut, MD,3 \\ ${ }^{1}$ Division of Maternal Fetal Medicine, West VIrginia University, \\ Morgantown, West Virginia \\ ${ }^{2}$ Duke University School of Medicine, Durham, North Carolina \\ ${ }^{3}$ Division of Maternal Fetal Medicine, Duke University, Durham, \\ North Carolina \\ Am J Perinatol Rep 2021;11:e34-e37. \\ Address for correspondence Megan Varvoutis, MD, Medical Center \\ Drive, PO Box 9186, Morgantown, WV 26506, United States \\ (e-mail: megvarvoutis@gmail.com).
}

\begin{abstract}
Keywords

- hysterectomy

- broad ligament

- spontaneous hematoma

- sepsis

Background Broad ligament hematomas are rare in the setting of vaginal delivery. When they do occur, patients typically present with acute hemodynamic instability. No cases of infected broad ligament hematomas have been reported.

Case A 22-year-old G2 P1011 status post vaginal delivery complicated by chorioamnionitis and pre-eclampsia presented 5 days postpartum with subjective complaints of fever, vomiting, and increased vaginal bleeding. She was treated with antibiotics and uterine evacuation was planned for presumed retained products. After dilation and curettage, the patient was transferred to our facility, as her clinical status did not improve, and was later found to have an infected broad ligament hematoma requiring hysterectomy.

Conclusion Though uncommon, broad ligament hematomas should be considered in postpartum women presenting with anemia and vaginal bleeding, even without hemodynamic instability. Recent intrauterine infections may predispose to hematoma infection.
\end{abstract}

\section{Teaching Objectives}

1. Describe the clinical presentation for spontaneous broad ligament hematoma after labor.

2. Describe the recommended medical therapy for spontaneous broad ligament hematoma.

Spontaneous broad ligament hematoma formation occurring during labor is exceedingly rare. Broad ligament hematomas are a known complication during cesarean delivery but spontaneous hematomas during labor or vaginal delivery are uncommon. Spontaneous broad ligament hematomas may result from spontaneous rupture of either branches of the uterine artery or uterine veins and are considered obstetric emergencies resulting in maternal mortality rates

received

March 15, 2020

accepted

May 21, 2020 as high as $40 \%$ when associated with labor. ${ }^{1}$ While broad ligament hematomas are a documented consequence of uterine artery or vein rupture, this report is unique because our patient's hematoma was complicated by delayed sepsis and anemia instead of acute hemodynamic changes. This case emphasizes the importance of early recognition and evacuation of infected hematomas to ensure the highest possibility of uterine preservation.

\section{Case Report}

We report a case of a 22-year-old G2 P1011 woman who underwent an uncomplicated spontaneous vaginal delivery at 41 weeks and 2 days who subsequently developed sepsis in the setting of a spontaneous broad ligament hematoma. She was

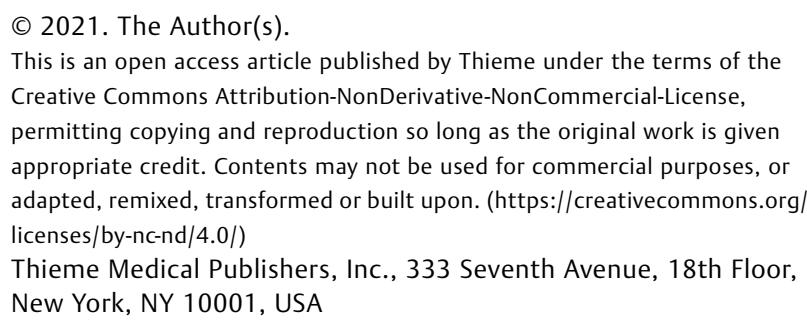


admitted to an outside hospital for an induction of labor at term. Her medical history was significant for class 1 obesity, and she had no history of prior uterine or pelvic surgery. Her cervix was unfavorable and she received a combination of misoprostol and Foley balloon catheter for cervical ripening and intravenous oxytocin for labor induction. The intrapartum course was complicated by chorioamnionitis and pre-eclampsia without severe features. The patient received an epidural for anesthesia and antibiotic therapy for chorioamnionitis was initiated. During the delivery admission, her hemoglobin was noted to be $11.7 \mathrm{~g} / \mathrm{dL}$ at the start of her induction, and there was no objective evidence of hemodynamic instability or abdominal pain during or following the delivery. Her labor lasted $\sim 40$ hours and resulted in a spontaneous vaginal delivery of a $3,870 \mathrm{~g}$ infant with a quantitative blood loss of $730 \mathrm{~mL}$. Her postpartum course was uneventful. She remained afebrile and hemodynamically stable and was discharged on the second postpartum day.

The patient then presented to the emergency room 5 days after delivery reporting a 1-day history of fever, nausea, and vomiting, and increased vaginal bleeding. Her initial vital signs were significant for a temperature of $39.5^{\circ} \mathrm{C}$, pulse of 144 beats per minute (bpm), and blood pressure of 144/89 mm Hg. Her abdominal exam was significant for exquisite fundal tenderness at the umbilicus, and her pelvic exam revealed a 20-week size uterus and a small amount of clotted blood in the vaginal vault consistent with normal postpartum lochia. The patient's complete blood count was notable for leukocytosis with a white blood count of $17.7 \times 10^{9} / \mathrm{L}$, anemia with a hemoglobin of $8.4 \mathrm{~g} / \mathrm{dL}$, and a venous lactate of $0.9 \mathrm{mmol} / \mathrm{L}$. Urine analysis results suggested probable contamination, and urine culture showed mixed flora.

An abdominal ultrasound demonstrated a thickened endometrial stripe with some flow signal (-Fig. 1A) and a heterogenous mass in the left pelvis inseparable from left uterus, favoring an exophytic uterine mass such as fibroid (-Fig. 1B). The working diagnosis at the time was concerning for endometritis and possible retained products of conception, and she was started on ertapenem and admitted for further monitoring.

After initiation of intravenous antibiotics, the patient became afebrile during the first 24 hours of that admission. However, on hospital day 2, she developed a fever again to $39.5^{\circ} \mathrm{C}$, so she was transitioned to meropenem. On hospital day 3 , she was noted to have persistent fever, moderate lochia, worsening tachycardia, leukocytosis, and anemia with a decrease in her hemoglobin to $7.0 \mathrm{~g} / \mathrm{dL}$, respectively. Hemolysis workup revealed normal haptoglobin and mildly elevated reticulocytes. At that point, blood cultures were ordered and clindamycin was added for group A streptococcus coverage.

Due to concern for retained products possibly being the cause of infection and fall in her hemoglobin levels, the decision was made to proceed to the operating room for an ultrasoundguided dilation and curettage $(D \& C)$. During the $D \& C$, scant filmy tissue and a small blood clot were aspirated from the uterine cavity. Despite multiple attempts with sharp curettage, further material was unable to be evacuated. Given the patient's worsening clinical status, inconsistent imaging and difficult $\mathrm{D} \& \mathrm{C}$ revealing minimal to no obvious retained products of conception, the patient was transferred to our tertiary care center for higher level of care.

Upon arrival to our facility, the patient's vitals were concerning for sepsis with a fever to $39.2^{\circ} \mathrm{C}$, tachycardia with pulse of $114 \mathrm{bpm}$, and new oxygen desaturations measured by pulse oximetry that required $8 \mathrm{~L} /$ minute oxygen supplementation by nasal canula. Abdominal exam at that time noted significant tenderness to palpation in the right lower quadrant. Pelvic exam revealed no active bleeding. Laboratory findings were significant for persistent leukocytosis and anemia, with lactate and a comprehensive metabolic panel was within normal limits. Chest X-ray demonstrated mild pulmonary edema and trace pleural effusions.

Repeat bedside ultrasonographic assessment revealed that the large heterogeneous mass in the patient's right adnexa was most consistent with a hematoma. Conversely, the mass in the patient's left pelvis was identified to be the uterus, displaced to the left by the hematoma. The contrasted computed tomography demonstrated a large hemorrhagic collection in the pelvis abutting the uterus concerning for potential uterine rupture with a large pelvic hematoma. In addition, significant right sided hydronephrosis was also present. (-Fig. 1C). Definitive extravasation of blood was not identified.

The patient was consented and scheduled for an emergent exploratory laparotomy for evacuation of hematoma, possible repair of ruptured uterus, and possible hysterectomy.

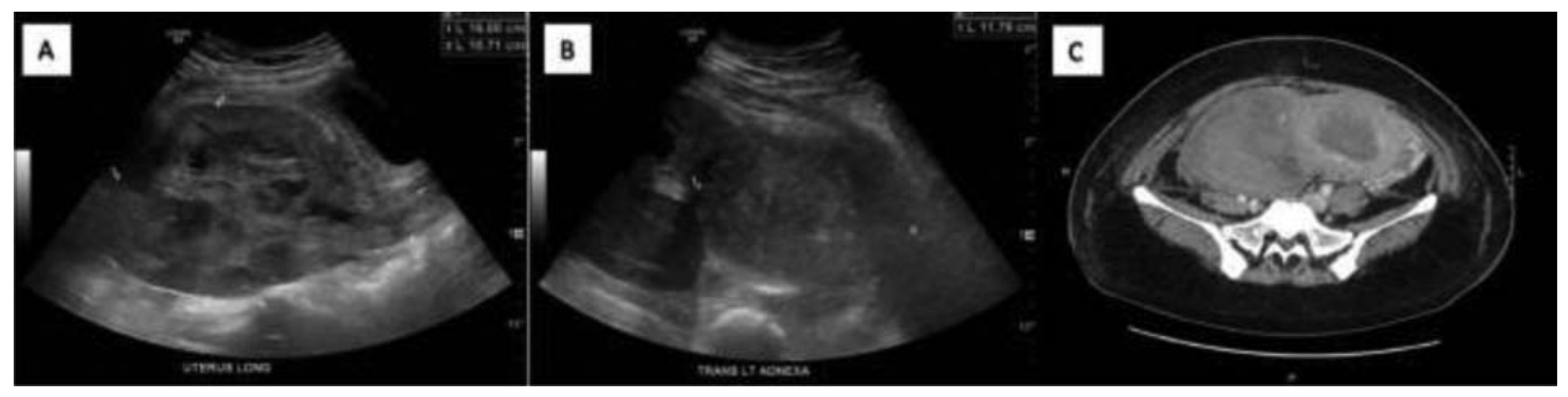

Fig. 1 (A) Heterogeneous mass measuring $16 \times 18.7 \times 10.7 \mathrm{~cm}$, with poorly defined cavity and thickened endometrial stripe, concerning for retained products and/or superimposed infection. (B) Heterogeneous mass in left pelvis inseparable from presumed uterus that was initially thought to represent an exophytic uterine mass such as fibroid. (C) Computed tomography abdomen and pelvis with large hemorrhagic collection in the right pelvis abutting an enlarged and irregularly shaped uterus on the left, concerning for uterine rupture. 


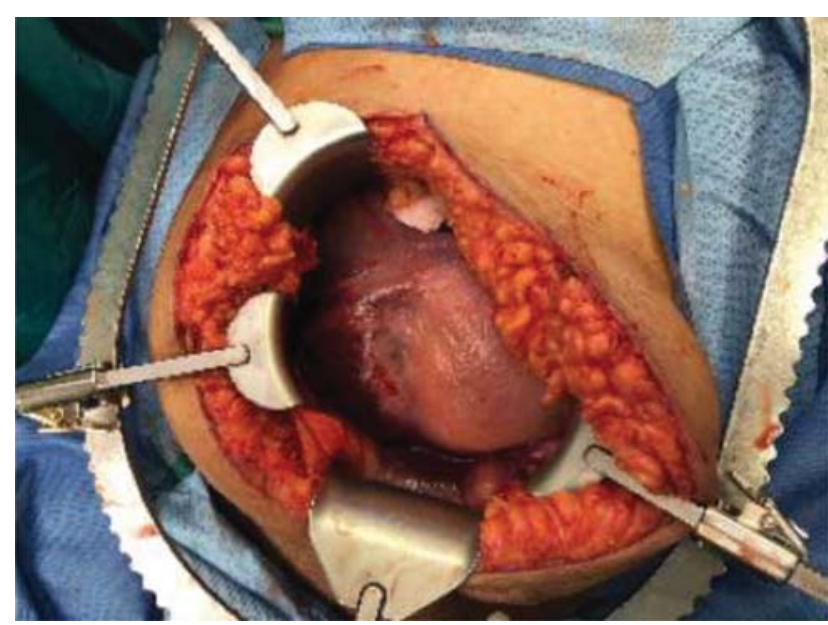

Fig. 2 Intraoperative broad ligament hematoma $(15 \times 15 \mathrm{~cm})$.

The patient received meropenem and cefazolin preoperatively. Initial abdominal survey demonstrated an enlarged discolored uterus with a $15 \times 15 \mathrm{~cm}$ right broad ligament hematoma extending into the retroperitoneum to the level of the pelvic brim (-Fig. 2). The uterine wall in the right lateral uterine segment was friable with weakened tissue integrity upon handling. There was noted to be a large defect in the broad ligament adjacent to where the cervical branch of the right uterine artery would have been. It was suspected that the hematoma was due to spontaneous rupture of the uterine artery in this region that subsequently bled into the broad ligament and retroperitoneal space. The remainder of the abdominal survey did not reveal any other abnormalities.

The blood and clot were evacuated from the hematoma, and no obvious actively bleeding vessel or uterine wall defect was noted. Given the patient's clinical status that suggested a systemic infection and the dusky appearance and friability of the uterus, the decision was made to proceed with a supracervical hysterectomy. In addition, a right ureteral stent was placed by urology intraoperatively for the severe hydroureteronephrosis. Estimated intraoperative blood loss was $550 \mathrm{~mL}$ including evacuated blood from the hematoma. She received three units of packed red blood cells during the operation.

After the procedure, the patient was started on vancomycin and piperacillin-tazobactam. She remained afebrile with downtrending white blood cell count and stable hemoglobin for the remainder of the hospitalization. She was discharged on postoperative day 3 with a 7-day course of trimethoprim-sulfamethoxazole, metronidazole, and prophylactic enoxaparin.

Final pathology demonstrated a large organizing hematoma involving the full thickness of the myometrium with acute and chronic inflammation without an identifiable uterine rupture and extensive myometrial necrosis. The patient was followed up in the obstetrics clinic for staple removal and was found to be doing well. Her right ureteral stent was removed in urology clinic 6 weeks following the procedure and repeat imaging demonstrated resolution of the hydronephrosis.

\section{Comment}

Broad ligament hematomas are rare in obstetrics, with an incidence of $\sim 1: 20,000,{ }^{2}$ and may occur as a result of operative vaginal or cesarean deliveries or spontaneously due to uterine artery or varices rupture. These hematomas typically form due to vaginal, cervical, or uterine tears extending to the uterine or vaginal arteries. It has been suggested that rapid labor, cesarean section, instrumental deliveries, and trauma may predispose patients to developing these lacerations. ${ }^{3}$ When broad ligament hematomas occur spontaneously, they are usually due to vessel rupture, most commonly the uterine artery. Spontaneous uterine artery rupture has been found to cause $40 \%$ maternal mortality when associated with labor and may be a result of pronounced blood pressure fluctuations during labor. ${ }^{1}$ Hormonal changes during pregnancy may also play a role as well-previous studies have shown that pregnancy-level estrogen, and to a lesser extent progesterone, suppresses intimal proliferation in response to vascular injury, ${ }^{4}$ putting vessels at greater risk of rupture. In the majority of cases, patients who developed broad ligament hematomas during labor present with acute hemodynamic instability within hours of delivery. ${ }^{2,5,6}$ There have not been any cases reported of broad ligament hematomas complicated by infection.

This case is unique because of our patient's delayed presentation as well the development of an infection progressing to sepsis. Our patient's relatively uneventful vaginal delivery without the need for instrumental assistance along with the intra-operative findings suggests that the cause of her hematoma was due to a spontaneous vessel rupture, likely the uterine artery, which probably occurred during labor or delivery. Her lack of hemodynamic instability suggests that the broad ligament was able to sufficiently contain the resulting hemorrhage and minimize the rate of extravasation, resulting in a delayed presentation. Because red blood cell levels may take up to days to reflect blood loss, diagnosing occult bleeds such as this becomes difficult without suggestive vital sign abnormalities. Though our patient became anemic after her delivery, her hemoglobin drop was appropriate for her quantitative blood loss. Nonetheless, anemia in the postpartum period should carefully evaluated for possible signs of intra-abdominal bleed.

By the time, the patient presented on postpartum day 5 , the hematoma had developed to a significant size and had also become infected. It is possible that her peripartum diagnosis of chorioamnionitis increased her risk for developing this infection, as previous studies have shown chorioamnionitis to be associated with increased risk of endomyometritis and pelvic abscesses despite appropriate treatment. ${ }^{7}$ Her initial ultrasound findings also noted a flow signal in what was later identified as the hematoma, suggesting a potential for active bleeding.

By the time the patient presented to the tertiary care center, the patient's status worsened and she had developed signs of sepsis. Due to her worsening clinical status and 
uncertain source of bleeding, she required laparotomy for removal of the source of infection. It is conceivable that if the infected hematoma had been identified earlier prior to her development of sepsis, it may have been possible for the patient to undergo only surgical evacuation of the hematoma, sparing the uterus. Early recognition and hematoma evacuation may have provided greater possibility of uterine preservation in this patient. If the hematoma had been recognized prior to the onset of infection, the patient may have also qualified for more conservative treatments such as uterine arterial embolization. ${ }^{3}$ However, because the patient was septic by the time the hematoma was discovered, a laparotomy with hematoma evacuation and hysterectomy was necessary to ensure that the source of infection was adequately removed.

Overall, this case emphasizes the management of a broad ligament hematoma, an obstetric emergency, on the differential for anemia and vaginal bleeding among intra- and postpartum patients. While these cases typically present with hemodynamic instability within hours after delivery, it is also possible for these bleeding episodes to occur at a slower rate without suggestive vital sign abnormalities. Slow changes in red blood cell counts can make this a difficult diagnosis, so any decrease in red blood counts after delivery should be carefully considered. Further, collections of blood in the setting of a recent intrauterine infection may predispose patients to developing a superimposed infection of the blood collection. While it is uncertain whether the uterus can be preserved in these cases, earlier diagnosis and drainage would likely increase the possibility.

\section{Funding}

The authors have no relevant financial disclosures to disclose as no funding was used from a commercial organization.

Conflict of Interest

None.

\section{References}

1 Ziereisen V, Bellens B, Gérard C, Baeyens L. [Spontaneous rupture of utero-ovarian vessels in postpartal period: a case report and review of the literature]. J Gynecol Obstet Biol Reprod (Paris) 2003;32(01):51-54

2 Saleem N, Ali HS, Irfan A, Afzal B. Broad ligament hematoma following a vaginal delivery in primigravida. Pak J Med Sci 2009; 25(04):683-685

3 Kovo M, Eshed I, Malinger G. Broad ligament hematoma following a normal vaginal delivery. Gynecol Surg 2006;3(02):138-140

4 Zhang L, Fishman MC, Huang PL. Estrogen mediates the protective effects of pregnancy and chorionic gonadotropin in a mouse model of vascular injury. Arterioscler Thromb Vasc Biol 1999; 19(09):2059-2065

5 Lee HJ, Na ED, Kim HC, Kim MS, Moon MJ. Massive broad ligament hematoma caused by spontaneous uterine artery rupture after uncomplicated vaginal delivery. J Minim Invasive Gynecol 2020; 27(02):250-253

6 Maxwell C, Gawler D, Green J. An unusual case of acute postpartum broad ligament haematoma. Aust N Z J Obstet Gynaecol 1997; 37(02):239-241

7 Rouse DJ, Landon M, Leveno KJ, et al;National Institute of Child Health And Human Development, Maternal-Fetal Medicine Units Network. The Maternal-Fetal Medicine Units cesarean registry: chorioamnionitis at term and its duration-relationship to outcomes. Am J Obstet Gynecol 2004;191(01):211-216 